\title{
Consistent tangent operators for constitutive rate equations
}

\author{
Wolfgang Fellin* and Alexander Ostermann ${ }^{\dagger}$
}

\author{
11 June 2002 \\ post-print of: \\ FELLIN, W.; OSTERMANN, A. (2002): Consistent Tangent Operators For \\ Constitutive Rate-Equations. In: International Journal for Numerical and Analytical \\ Methods in Geomechanics 26/12, pp. 1213 - 1233.
}

\begin{abstract}
SUMMARY
A general approach for obtaining the consistent tangent operator for constitutive rate equations is presented. The rate equations can be solved numerically by the user's favorite time integrator. In order to obtain reliable results, the substepping in integration should be based on a control of the local error. The main ingredient of the consistent tangent operator, namely the derivative of the stress with respect to the strain increment must be computed simultaneously with the same integrator, applied to a numerical approximation of the variational equations. This information enables finite-element packages to assemble a consistent tangent operator and thus guarantees quadratic convergence of the equilibrium iterations. Several numerical examples with a hypoplastic constitutive law are given. As numerical integrator we used a second-order extrapolated Euler method. Quadratic convergence of the equilibrium iteration is shown.
\end{abstract}

KEY WORDS: consistent tangent operators; rate equations; hypoplasticity; time integration; error control; equilibrium iteration

\section{Introduction}

For the efficient simulation of complex problems in computational inelasticity, consistent tangent operators are, without doubt, of crucial importance [1]. It is well known that any inconsistency with the stress-update algorithm of the constitutive law will spoil the quadratic convergence of Newton's method in the iterative solution of the initial-boundary value problem. A consequence will be computational inefficiency.

\footnotetext{
${ }^{*}$ Universität Innsbruck, Institut für Geotechnik und Tunnelbau, Technikerstr. 13, A-6020 Innsbruck, Austria, e-mail: wolfgang.fellin@uibk.ac.at

${ }^{\dagger}$ Universität Innsbruck, Institut für Technische Mathematik, Geometrie und Bauinformatik, Technikerstr. 13, A-6020 Innsbruck, Austria, e-mail: alexander.ostermann@uibk.ac.at
} 
For simple constitutive laws, the consistent tangent operator can by found analytically by differentiation. For more complicated constitutive laws, however, this can be a tedious task and it is sometimes even not feasible. A remedy is then numerical differentiation. To our knowledge, one of the first papers that considered numerical differentiation as an appropriate approach was that of Miehe [2]. For computational plasticity, this idea has been further developed in a series of papers by Pérez-Foguet et al. $[3,4,5]$.

The main goal of the present paper is to explain how an efficient, accurate and robust approximation of the consistent tangent operator can be obtained for constitutive equations in rate form. Our framework is based on the variational equations and their approximation by finite differences. This approach is quite general and can be used in combination with any time integrator. Moreover, we use an adaptive step size strategy that is based on the control of the local integration error. In our opinion such an error control is indispensable for complex problems, since it is the only way to achieve adaptivity.

For the sake of simplicity, we present our ideas with the help of the constitutive models in hypoplasticity. We emphasize, however, that our approach is sufficiently general to cover all constitutive laws in rate form such as the CloE-model [6].

One of the first finite-element implementations of hypoplasticity dates back to Hügel [7]. For time integration he used the explicit Euler method with constant step sizes. The errors are not controlled during integration, however, the user can define limits for the maximum step size. The tangent operator is approximated by the material stiffness at the end of the load increment. An more advanced implementation was made by Roddeman [8]. He also used the forward Euler method, but adjusted the step size due to a rough error estimate. He further introduced an appropriate handling of the admissible states. The tangent operator is approximated by numerical differentiation of the constitutive law at the end of the load increment. In a recent work by Heeres [9], the backward Euler method is used for time integration. He allows substepping and computes the tangent operator numerically, following the ideas of [3]. A precise error control, however, is not provided.

The present article is organized as follows. In Section 2 we shortly describe the constitutive equations in hypoplasticity. In Section 3, we discuss their time integration and we explain how the consistent tangent operator is obtained numerically. We further describe the error control of the time integration on which our adaptive step size strategy is based, and we give some implementational details. The subsequent two sections illustrate our approach with various numerical examples. In Section 4, we study numerical aspects of our implementation, whereas Section 5 is devoted to finite-element problems. Apart from simple element tests, we consider the deformation of a vertically compressed soil specimen that develops a shear band due to an initial imperfection. Our main conclusions are finally given in Section 6.

\section{Hypoplasticity}

Hypoplastic laws have been mainly developed to describe the stress-strain behaviour of sands. They represent the objective stress rate [10] of the effective Cauchy stress $\stackrel{\circ}{\mathbf{T}}$ as a function of the effective Cauchy stress $\mathbf{T}$, the Eulerian stretching $\mathbf{D}$ and some additional state variables

$$
\stackrel{\circ}{\mathbf{T}}=\mathbf{h}(\mathbf{T}, \mathbf{D}, e) .
$$


In the version of von Wolffersdorff [11] used here, the additional state variable is the void ratio $e$ of the soil.

The function $\mathbf{h}$ is incrementally non-linear in $\mathbf{D}$ to capture the stiffness change in loading and unloading cycles. As the behaviour of sand is assumed to be approximately time independent, basic hypoplastic laws are time-scale invariant, thus $\mathbf{h}$ is positively homogeneous of the first degree in $\mathbf{D}$. Furthermore $\mathbf{h}$ is homogeneous in $\mathbf{T}$ in order to obtain proportional stress-paths in case of proportional strain-paths. For a more thorough discussion, we refer to the monograph of Kolymbas [12].

Since the mass is assumed to stay constant, the evolution of the void ratio $e$ is described by

$$
\dot{e}=(1+e) \operatorname{tr} \mathbf{D}=\mathbf{k}(\mathbf{T}, \mathbf{D}, e) .
$$

Note that $\mathbf{h}$ is not defined for $\operatorname{tr} \mathbf{T} \geq 0$, and that $e$ is bounded between the pressure dependent upper limit $e_{i}$ and the lower limit $e_{d}$, see Appendix A. This has to be considered in the time integration scheme.

Recent versions of hypoplasticity, e.g. hypoplasticity with intergranular strains [13], include further additional state variables. They can also be handled by our method.

\section{Time integration of the constitutive law}

The equations of motion together with the constitutive law form a coupled system consisting of an initial-boundary value problem and an ordinary differential equation. A steady state solution of this system is usually obtained by co-simulation: the equations of motion are solved with the help of a finite-element package, and the constitutive law by a solver for ordinary differential equations. As an example, we describe henceforth the situation for ABAQUS ${ }^{1}$. However, the ideas presented below are not at all based on ABAQUS. The can be used with any co-simulation based finite-element package. The relevant constitutive information is passed to ABAQUS by a subroutine UMAT which has to be supplied by the user. Starting from an equilibrium at time $t_{a}$, ABAQUS performs an (incremental) loading and provides the subroutine UMAT with the Cauchy stress tensor $\boldsymbol{\sigma}\left(t_{a}\right)$ at the beginning of the loading as well as with the time increment $\Delta t$ and an initial guess $\Delta \varepsilon^{0}$ for the strain increment [14]. The subroutine UMAT has then to supply ABAQUS with the new Cauchy stress tensor $\boldsymbol{\sigma}\left(t_{a}+\Delta t\right)$, updated according to the constitutive law as well as with the derivative of $\sigma$ with respect to the strain increment [15]. With this information, a new guess for the strain increment is calculated and the whole procedure is iterated until convergence. The precise information on the Jacobian

$$
\frac{\partial \Delta \boldsymbol{\sigma}}{\partial \Delta \varepsilon}=\frac{\partial \boldsymbol{\sigma}\left(t_{a}+\Delta t\right)}{\partial \Delta \varepsilon}
$$

is essential to achieve fast (quadratic) convergence in the Newton-type iteration performed by ABAQUS. With a poor approximation in (3), the speed of convergence can be very slow and might even demand $\Delta t$ to be very small too.

One problem of the co-simulation approach is that the equilibrium iterations performed by ABAQUS are decoupled from the stress computations over a time window of length $\Delta t$. Information between the

${ }^{1}$ ABAQUS is a trademark of HKS Inc., Hibbit, Karlson \& Sorenson, Rhode Island, USA 
the two subsystems is only exchanged at the beginning and at the end of this time window. Therefore, the temporal rate of the strain tensor is not known as a function of time. Only its mean value over the window

$$
\mathrm{D}=\frac{\Delta \varepsilon}{\Delta t}
$$

is available for use in the constitutive law. Moreover, since our constitutive law is path-dependent, the simulation results might slightly depend on the loading history, i.e. on the choice of $\Delta t$. This, of course, is rather a problem of the modeling than a problem of discretization. A remedy is to supply an upper bound on $\Delta t$ at the price of a higher computing time.

In the remainder of this section we will explain how to obtain the Jacobian (3) for constitutive laws in rate form. This Jacobian is necessary to compute the consistent tangent operator for the initialboundary value problem. Further we will describe an adaptive time integration of the constitutive equations. The sizes of the substeps are there controlled by an asymptotically correct local error estimator. Finally, we will give some implementational details.

\subsection{Consistent tangent operator}

For the given initial stress tensor $\mathbf{T}(0)=\boldsymbol{\sigma}\left(t_{a}\right)$ and a strain increment $\Delta \boldsymbol{\varepsilon}$, the subroutine UMAT has to provide the new stress tensor $\boldsymbol{\sigma}\left(t_{a}+\Delta t\right)=\mathbf{T}(\Delta t)$ at time $t_{a}+\Delta t$ as well as its derivative with respect to the strain increment. Note that for ABAQUS only the co-rotational parts have to be computed [15]. Thus we have to solve the following system of differential equations for $0 \leq t \leq \Delta t$

$$
\begin{aligned}
\frac{\mathrm{d}}{\mathrm{d} t} \mathbf{T} & =\mathbf{h}(\mathbf{T}, \mathbf{D}, \mathbf{Q}), & \mathbf{T}(0) & =\boldsymbol{\sigma}\left(t_{a}\right), \\
\frac{\mathrm{d}}{\mathrm{d} t} \mathbf{Q} & =\mathbf{k}(\mathbf{T}, \mathbf{D}, \mathbf{Q}), & \mathbf{Q}(0) & =\mathbf{Q}_{0} .
\end{aligned}
$$

Here, $\mathrm{Q}$ denotes the additional state variables, and $\mathrm{Q}_{0}$ are their values at time $t_{a}$. The hypoplastic law considered in this paper has only one additional state variable, denoted by $e$. In this case the function $\mathbf{k}$ is given by (2).

Differentiation of (5) with respect to $\mathbf{D}$ yields the variational equations

$$
\begin{array}{lll}
\frac{\mathrm{d}}{\mathrm{d} t} \frac{\partial \mathbf{T}}{\partial \mathbf{D}}=\frac{\partial \mathbf{h}}{\partial \mathbf{T}}(\mathbf{T}, \mathbf{D}, \mathbf{Q}) \cdot \frac{\partial \mathbf{T}}{\partial \mathbf{D}}+\frac{\partial \mathbf{h}}{\partial \mathbf{Q}}(\mathbf{T}, \mathbf{D}, \mathbf{Q}) \cdot \frac{\partial \mathbf{Q}}{\partial \mathbf{D}}+\frac{\partial \mathbf{h}}{\partial \mathbf{D}}(\mathbf{T}, \mathbf{D}, \mathbf{Q}), & \frac{\partial \mathbf{T}}{\partial \mathbf{D}}(0)=0, \\
\frac{\mathrm{d}}{\mathrm{d} t} \frac{\partial \mathbf{Q}}{\partial \mathbf{D}}=\frac{\partial \mathbf{k}}{\partial \mathbf{T}}(\mathbf{T}, \mathbf{D}, \mathbf{Q}) \cdot \frac{\partial \mathbf{T}}{\partial \mathbf{D}}+\frac{\partial \mathbf{k}}{\partial \mathbf{Q}}(\mathbf{T}, \mathbf{D}, \mathbf{Q}) \cdot \frac{\partial \mathbf{Q}}{\partial \mathbf{D}}+\frac{\partial \mathbf{k}}{\partial \mathbf{D}}(\mathbf{T}, \mathbf{D}, \mathbf{Q}), & \frac{\partial \mathbf{Q}}{\partial \mathbf{D}}(0)=0 .
\end{array}
$$

Let $\Delta \boldsymbol{\sigma}=\boldsymbol{\sigma}\left(t_{a}+\Delta t\right)-\boldsymbol{\sigma}\left(t_{a}\right)$. In order to get

$$
\frac{\partial \Delta \boldsymbol{\sigma}}{\partial \Delta \varepsilon}=\frac{\partial \boldsymbol{\sigma}\left(t_{a}+\Delta t\right)}{\partial \Delta \varepsilon}=\frac{1}{\Delta t} \cdot \frac{\partial \mathbf{T}}{\partial \mathbf{D}}(\Delta t),
$$

system (6) has to be solved simultaneously with system (5). Due to the complicated structure of our constitutive law, the calculation (and implementation) of the expressions appearing on the righthand side of (6) seems to be a tedious task. We therefore strongly recommend to replace (6) by the 
following approximation which is obtained by numerical differentiation

$$
\begin{array}{rlrl}
\frac{\mathrm{d}}{\mathrm{d} t} \mathbf{B}_{i j} & =\frac{1}{\vartheta}\left(\mathbf{h}\left(\mathbf{T}+\vartheta \mathbf{B}_{i j}, \mathbf{D}+\vartheta \mathbf{V}_{i j}, \mathbf{Q}+\vartheta \mathbf{G}_{i j}\right)-\mathbf{h}(\mathbf{T}, \mathbf{D}, \mathbf{Q})\right), & \mathbf{B}_{i j}(0)=0, \\
\frac{\mathrm{d}}{\mathrm{d} t} \mathbf{G}_{i j}=\frac{1}{\vartheta}\left(\mathbf{k}\left(\mathbf{T}+\vartheta \mathbf{B}_{i j}, \mathbf{D}+\vartheta \mathbf{V}_{i j}, \mathbf{Q}+\vartheta \mathbf{G}_{i j}\right)-\mathbf{k}(\mathbf{T}, \mathbf{D}, \mathbf{Q})\right), & \mathbf{G}_{i j}(0)=0,
\end{array}
$$

for $1 \leq i \leq j \leq 3$. Here, $\mathbf{V}_{i j}$ denotes the standard basis tensor

$$
\mathbf{V}_{i j}=\left(\delta_{i k} \delta_{j \ell}\right)_{k, \ell=1}^{3}
$$

with the Kronecker symbol

$$
\delta_{i k}= \begin{cases}1 & \text { if } i=k \\ 0 & \text { else }\end{cases}
$$

A Taylor expansion of the right-hand side of (7) shows that

$$
\mathbf{B}_{i j}=\frac{\partial \mathbf{T}}{\partial D_{i j}}+\mathcal{O}(\vartheta)
$$

Thus the six tensors $\mathbf{B}_{i j}$ are good approximations for $\vartheta$ sufficiently small. From the numerical point of view, the parameter $\vartheta$ should be chosen as

$$
\vartheta=\max (1,\|\mathbf{D}\|) \cdot \sqrt{\mathrm{EPS}},
$$

where EPS denotes the machine precision. In IEEE double precision arithmetic, we have

$$
\mathrm{EPS} \approx 10^{-16} .
$$

In the full 3-D case, (7a) has essentially 36 components whereas (7b) has another 6 components for every additional state variable. We propose to solve (5) and (7) simultaneously with the same numerical method (as described subsequently). This guarantees the consistency of the derivatives.

\subsection{Adaptive time integration}

In this subsection we will explain how to solve (5) and (7) numerically. In principle, any adaptive code can be used for this task. In our subroutine UMAT we have implemented a second-order method which derives from the forward Euler scheme. The error and step size control is based on Richardson extrapolation which proved to be robust and very reliable. Let us shortly explain this method. Collecting all the variables of our problem in a super-vector

$$
\begin{aligned}
\mathbf{y}= & \left(T_{11}, T_{22}, T_{33}, T_{12}, T_{13}, T_{23},\left(B_{11}\right)_{11},\left(B_{11}\right)_{22},\left(B_{11}\right)_{33},\left(B_{11}\right)_{12},\left(B_{11}\right)_{13},\left(B_{11}\right)_{23},\right. \\
& \left.\left(B_{22}\right)_{11}, \ldots,\left(B_{22}\right)_{23},\left(B_{33}\right)_{11}, \ldots,\left(B_{23}\right)_{23}, Q_{1}, \ldots, Q_{m},\left(G_{11}\right)_{1}, \ldots,\left(G_{11}\right)_{m},\left(G_{22}\right)_{1}, \ldots,\left(G_{23}\right)_{m}\right)^{T}
\end{aligned}
$$

and denoting the right-hand sides of (5) and (7) by $\mathbf{F}$, we have to solve the initial value problem

$$
\mathbf{y}^{\prime}(t)=\mathbf{F}(\mathbf{y}(t)), \quad \mathbf{y}(0)=\mathbf{y}_{0} \quad \text { given } .
$$


We choose an initial step size $\tau$ satisfying $0 \leq \tau \leq \Delta t$ where $\Delta t$ is the given increment by ABAQUS and calculate the two approximations

$$
\mathbf{v}=\mathbf{y}_{0}+\tau \mathbf{F}\left(\mathbf{y}_{0}\right)
$$

and

$$
\mathbf{w}=\mathbf{y}_{0}+\frac{\tau}{2} \mathbf{F}\left(\mathbf{y}_{0}\right)+\frac{\tau}{2} \mathbf{F}\left(\mathbf{y}_{0}+\frac{\tau}{2} \mathbf{F}\left(\mathbf{y}_{0}\right)\right)
$$

which need two evaluations of the right-hand side function $\mathbf{F}$. Whereas $\mathbf{v}$ is the result of the forward Euler method with step size $\tau, \mathbf{w}$ is the result of two Euler steps of size $\tau / 2$.

To study the error of these approximations after one step, we expand the exact solution into a Taylor series

$$
\mathbf{y}(\tau)=\mathbf{y}(0)+\tau \mathbf{y}^{\prime}(0)+\frac{\tau^{2}}{2} \mathbf{y}^{\prime \prime}(0)+\mathcal{O}\left(\tau^{3}\right)
$$

and use (8) to obtain

$$
\mathbf{y}(\tau)=\mathbf{y}_{0}+\tau \mathbf{F}\left(\mathbf{y}_{0}\right)+\frac{\tau^{2}}{2} \mathbf{F}^{\prime}\left(\mathbf{y}_{0}\right) \mathbf{F}\left(\mathbf{y}_{0}\right)+\mathcal{O}\left(\tau^{3}\right)
$$

This shows

$$
\mathbf{y}(\tau)-\mathbf{v}=\frac{\tau^{2}}{2} \mathbf{F}^{\prime}\left(\mathbf{y}_{0}\right) \mathbf{F}\left(\mathbf{y}_{0}\right)+\mathcal{O}\left(\tau^{3}\right)
$$

and

$$
\mathbf{y}(\tau)-\mathbf{w}=\frac{\tau^{2}}{4} \mathbf{F}^{\prime}\left(\mathbf{y}_{0}\right) \mathbf{F}\left(\mathbf{y}_{0}\right)+\mathcal{O}\left(\tau^{3}\right)
$$

Therefore, the difference

$$
\mathbf{w}-\mathbf{v}=\frac{\tau^{2}}{4} \mathbf{F}^{\prime}\left(\mathbf{y}_{0}\right) \mathbf{F}\left(\mathbf{y}_{0}\right)+\mathcal{O}\left(\tau^{3}\right)
$$

is an asymptotically correct estimate for the error. We set

$$
\mathrm{EST}=\|\mathbf{w}-\mathbf{v}\|
$$

and use the following step size strategy which is standard in this field, see [16].

If the estimated error EST is below the user-supplied tolerance TOL, the step is accepted and we use the extrapolated value

$$
\mathbf{y}_{\text {new }}=2 \mathbf{w}-\mathbf{v}
$$

which due to (9) is a second-order approximation to the solution. Further, we enlarge our step size accordingly to

$$
\tau_{\text {new }}=\tau \cdot \min \left(5,0.9 \cdot \sqrt{\frac{\mathrm{TOL}}{\mathrm{EST}}}\right),
$$

which would have been a proper step size for the present step. We then continue our integration until we reach $\Delta t$.

If the estimated error EST is larger than TOL, we have to reject the step and to redo it with a smaller step size given by

$$
\tau_{\text {new }}=\tau \cdot \max \left(0.2,0.9 \cdot \sqrt{\frac{\mathrm{TOL}}{\mathrm{EST}}}\right) .
$$

The factor 0.9 is a safety factor. The other two constants in (11) limit the maximum step size change to a factor of 5. For these three constants we have chosen the standard values from literature, see [16]. 


\subsection{Implementation}

We have implemented the above strategy in a FORTRAN subroutine UMAT for ABAQUS. This subroutine is freely available on the Web [17]. Let us describe in this section some implementational details.

In order to make the error estimation (10) more flexible, we have used a weighted Euclidean norm over the stress variables

$$
\|\mathbf{w}-\mathbf{v}\|=\sqrt{\sum_{i=1}^{6}\left(\frac{w_{i}-v_{i}}{s_{i}}\right)^{2}}
$$

with the solution depending weights

$$
s_{i}=\max \left(y_{i}, w_{i}\right)+\text { ATOL } .
$$

The threshold value ATOL can be supplied by the user. Its default value is ATOL $=10^{-3}$.

The constitutive law is possibly not defined for all values of the state variables. For example hypoplasticity is not defined for $\operatorname{tr} \mathbf{T} \geq 0$ and $\mathbf{Q}=e<e_{d}$, see appendix A. Using a forward time integration one can reach such an inadmissible state at the end of the time step $t+\tau$. This means that $\mathbf{F}\left(\mathbf{y}_{\text {new }}\right)$ can not be evaluated. If this happens the step is rejected and done again with half of the step size. This strategy was also used by $[8,18]$. If the new step size is less than $10^{-10}$ the time integration is interrupted and ABAQUS is asked to reduce its load increment $\Delta t$ by a factor of 4 .

\section{An illustrative academic example}

First we treat a simple but illustrative example to study some numerical aspects of our implementation. We assume small strains and use a simplified one-dimensional hypoplastic law to simulate loading in a one-dimensional compression test [19]

$$
\dot{T}=K T \dot{\varepsilon},
$$

with the constant $K=-2000$ and an initial stress $T_{0}=-100 \mathrm{~N} / \mathrm{m}^{2}$. The loading is $\bar{T}=-1000 \mathrm{~N} / \mathrm{m}^{2}$. The initial-boundary value problem to be solved reduces to

$$
T(\varepsilon)-\bar{T}=0 \text {. }
$$

The analytic solution of problem (13), (12) is given by

$$
\varepsilon^{\mathrm{an}}=\frac{1}{K} \ln \frac{\bar{T}}{T_{0}}=-1.1512 \times 10^{-3} .
$$

Equation (13) was also solved with the standard Newton method

$$
\varepsilon^{i+1}=\varepsilon^{i}-\left(\frac{\mathrm{d} T^{i}}{\mathrm{~d} \varepsilon^{i}}\right)^{-1} R^{i},
$$


with the residual $R$ in each iteration $i$

$$
R^{i}=T\left(\varepsilon^{i}\right)-\bar{T}
$$

The consistent tangent $\mathrm{d} T^{i} / \mathrm{d} \varepsilon^{i}$ is calculated together with the numerical stress $T^{i}=T\left(\varepsilon^{i}\right)$ during the integration of (12) with the above described scheme. As starting value $\varepsilon^{0}=10^{-3}$ was chosen.

Several calculations with different time integration schemes were done in IEEE quadruple precision. To compare the numerical results $\varepsilon^{i}$ with the analytical solution (14), the relative error

$$
\operatorname{err} \varepsilon=\frac{\varepsilon^{i}-\varepsilon^{\text {an }}}{\varepsilon^{\text {an }}}
$$

is used. To show the accuracy of the time integration, the analytical stress $T^{\text {an }}$ is evaluated for the numerical strain of the last iteration

$$
T^{\mathrm{an}}=T_{0} e^{K \varepsilon^{i}}
$$

and compared with the numerical stress $T^{i}$

$$
\operatorname{err} T=\frac{T^{i}-T^{\mathrm{an}}}{T^{\mathrm{an}}}
$$

The results are displayed in Tables 1 and 2 .

Table 1. Newton iteration of (13) with forward Euler time integration of (12) and constant time step sizes.

\begin{tabular}{|c|c|}
\hline \multicolumn{2}{|c|}{1 Euler step } \\
\hline It.No. & $R^{i}$ \\
\hline 1 & 700.0 \\
2 & $-3.881 \times 10^{-19}$ \\
3 & $1.262 \times 10^{-29}$ \\
& \\
& \\
\hline err $\varepsilon$ & -2.908 \\
\hline \hline err $T$ & 0.998 \\
\hline
\end{tabular}

\begin{tabular}{|c|c|}
\hline \multicolumn{2}{|c|}{ 10 Euler steps } \\
\hline It.No. & $R^{i}$ \\
\hline 1 & 380.8 \\
2 & -124.6 \\
3 & -6.040 \\
4 & $-1.629 \times 10^{-2}$ \\
5 & $-1.195 \times 10^{-7}$ \\
6 & $-5.573 \times 10^{-18}$ \\
\hline err $\varepsilon$ & -0.124 \\
\hline \hline err $T$ & 0.2492 \\
\hline
\end{tabular}

\begin{tabular}{|c|c|}
\hline \multicolumn{2}{|c|}{1000 Euler steps } \\
\hline It.No. & $R^{i}$ \\
\hline 1 & 262.5 \\
2 & -52.75 \\
3 & -1.298 \\
4 & $-8.412 \times 10^{-4}$ \\
5 & $-3.534 \times 10^{-10}$ \\
6 & $4.985 \times 10^{-21}$ \\
\hline err $\varepsilon$ & $-1.152 \times 10^{-3}$ \\
\hline \hline err $T$ & $2.649 \times 10^{-3}$ \\
\hline
\end{tabular}

For all calculations with constant step sizes in time integration, the overall Newton method converges quadratically, although the solutions for one and 10 substeps are very inaccurate, see Table 1 . Thus it is very important to control the error of the time integration scheme.

The situation is slightly different for adaptive step sizes. If the constitutive equations are integrated with an accuracy of TOL $=10^{-3}$, which is a reasonable choice in engineering applications, quadratic convergence is lost. Nevertheless the overall convergence is very fast, see Table 2-left, and the final solution has the required accuracy. On the other hand, if the constitutive law is integrated with a very high precision such as TOL $=10^{-10}$, quadratic convergence shows up again, see Table 2-right.

This behaviour can be explained as follows. The function $R^{\text {an }}$ is approximated by numerical integration. This approximation $R$ is in fact a composition of formulas (e.g. forward Euler steps) that 
Table 2. Newton iteration of (13) with extrapolated Euler time integration of (12), adapted time steps and error control.

\begin{tabular}{|c|c|}
\hline \multicolumn{2}{|c|}{ Extrapolated Euler, TOL $=10^{-3}$} \\
\hline It.No. & $R^{i}$ \\
\hline 1 & 261.9 \\
2 & -52.90 \\
3 & -1.249 \\
4 & $-7.414 \times 10^{-4}$ \\
5 & $-1.102 \times 10^{-5}$ \\
6 & $-1.703 \times 10^{-9}$ \\
\hline err $\varepsilon$ & $-5.388 \times 10^{-4}$ \\
\hline \hline err $T$ & $1.239 \times 10^{-3}$ \\
\hline
\end{tabular}

\begin{tabular}{|c|c|}
\hline \multicolumn{2}{|c|}{ Extrapolated Euler, TOL $=10^{-10}$} \\
\hline It.No. & $R^{i}$ \\
\hline 1 & 261.1 \\
2 & -52.07 \\
3 & -1.267 \\
4 & $-8.024 \times 10^{-4}$ \\
5 & $-3.218 \times 10^{-10}$ \\
6 & $4.629 \times 10^{-20}$ \\
\hline err $\varepsilon$ & $-5.400 \times 10^{-11}$ \\
\hline \hline err $T$ & $1.243 \times 10^{-10}$ \\
\hline
\end{tabular}

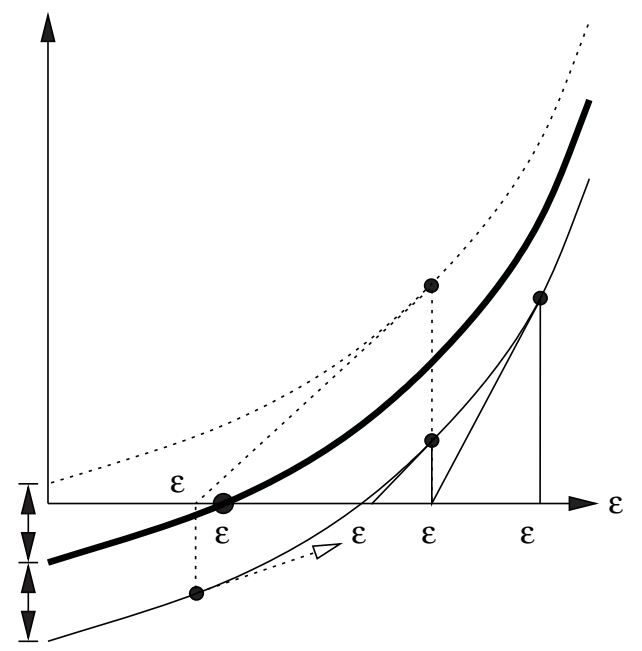

Figure 1. Newton iteration with inexact evaluation of the residuum.

depends on the chosen step size sequence. Therefore we might change the problem, i.e. switch from $R$ to a neighbouring $\widetilde{R}$, whenever changing the step size sequence from one Newton iteration to the other due to the error control of the time integrator. Such a behavior is illustrated in Figure 1 by the sequence $\varepsilon^{0}, \varepsilon^{1}, \tilde{\varepsilon}^{2}$, etc. The iteration might thus become quite irregular whenever the numerical solution is very close to the exact one. However, this is no problem at all since such a numerical solution is accurate enough anyway and should therefore be accepted. Only if the step size sequence is kept fixed for all Newton iterations, we stay on the same numerical approximation $R$ and thus quadratic convergence to the zero of $R$ is achieved, see Figure 1. Nevertheless, this solution is not better than the previous one, since it is only a zero of $R$ which in general is different from $\varepsilon^{\text {an }}$, see Table 1

As a last example, we show quadratic convergence for the above example using the hypoplastic law (20) with the parameters given in Table 11. The results are displayed in Table 3. For residuals larger than then the accuracy TOL $=10^{-10}$ of the stresses , quadratic convergence is obtained. 
Table 3. Newton iteration of (13) with extrapolated Euler time integration of the hypoplastic constitutive equation (20), adapted time steps and error control.

\begin{tabular}{|c|c|}
\hline \multicolumn{2}{|c|}{ Extrapolated Euler, TOL $=10^{-10}$} \\
\hline It.No. & $R^{i}$ \\
\hline 1 & 150.4 \\
2 & -7.369 \\
3 & $-1.482 \times 10^{-2}$ \\
4 & $-6.042 \times 10^{-8}$ \\
5 & $1.461 \times 10^{-19}$ \\
6 & $6.248 \times 10^{-27}$ \\
\hline$\varepsilon$ & $-1.1231 \times 10^{-3}$ \\
\hline
\end{tabular}

\section{Finite-element examples}

The performance of the proposed time integration scheme is shown with some element tests and with a shear band calculation. The computations were performed on a Silicon Graphics o200 with ABAQUS 5.8-10. Plane strain 4-node bilinear solid elements were used.

Unless otherwise stated we take large strains into account, we use the default convergence criteria and load incrementation of ABAQUS, and we set the error tolerances in the constitutive time integration to $\mathrm{TOL}=10^{-3}$ (relative error) and ATOL $=10^{-3}$ (absolute error).

The results are compared with an implementation of the von Wolffersdorff hypoplastic law by Roddeman [8], which is very robust and provides a relatively cheap but only rough approximation of the Jacobian. Therefore the convergence of the global system is quite slow.

In hypoplastic examples we use the parameters given in Table 11 of Appendix A.

\subsection{One-dimensional compression}

We first investigate the one-dimensional compression test, given in Figure 2. The initial conditions are $T_{22}=-p_{2}=-100 \mathrm{~N} / \mathrm{m}^{2}, T_{11}=-50 \mathrm{~N} / \mathrm{m}^{2}$, the initial void ratio is $e=0.735$. The loading is done by increasing the top pressure to $p_{2}=1000 \mathrm{~N} / \mathrm{m}^{2}$ in one increment.

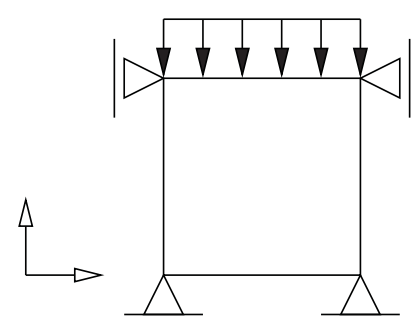

Figure 2. One-dimensional compression element test.

To show the numerical performance of ABAQUS the one-dimensional compression test is first solved with the simplified one-dimensional hypoplastic law (12). So we can compare the convergence of the 
equilibrium iterations of ABAQUS in Table 4 with the convergence in Tables 1 and 2. Note that the residuals in Tables 1 and 2 are stresses, and the largest residual force in Table 4 is the force in one node of the element with a cross section area of $1 \mathrm{~m}^{2}$. Thus they differ by a factor of 2 .

Table 4. Equilibrium iteration in ABAQUS using the constitutive equation (12). In contrast to Tables 1 and 2 the variables are in double precision only.

\begin{tabular}{|c|c|c|c|c|c|}
\hline \multicolumn{6}{|c|}{ Equilibrium iteration in ABAQUS: Largest residual force } \\
\hline \multirow[b]{2}{*}{ It.No. } & \multicolumn{3}{|c|}{ small strains } & \multicolumn{2}{|c|}{ large strains } \\
\hline & $\begin{array}{c}\text { Forward Euler } \\
1000 \text { steps }\end{array}$ & $\begin{array}{c}\text { Extrapolated Euler } \\
\text { TOL }=10^{-3}\end{array}$ & $\begin{array}{l}\text { Extrap. Euler } \\
\text { TOL }=10^{-10}\end{array}$ & $\begin{array}{c}\text { Forward Euler } \\
1000 \text { steps }\end{array}$ & $\begin{array}{l}\text { Extrap. Euler } \\
\text { TOL }=10^{-10}\end{array}$ \\
\hline 1 & -131 & -131 & -131 & -131 & -130 \\
\hline 2 & 26.4 & 26.4 & 26.0 & 26.2 & 25.8 \\
\hline 3 & 0.649 & 0.628 & 0.634 & 0.652 & 0.636 \\
\hline 4 & $4.225 \times 10^{-4}$ & $-5.092 \times 10^{-4}$ & $4.033 \times 10^{-4}$ & $7.517 \times 10^{-4}$ & $7.240 \times 10^{-4}$ \\
\hline 5 & $1.666 \times 10^{-9}$ & $7.384 \times 10^{-7}$ & $1.401 \times 10^{-9}$ & $3.798 \times 10^{-7}$ & $3.648 \times 10^{-7}$ \\
\hline 6 & $1.023 \times 10^{-12}$ & $-1.068 \times 10^{-9}$ & $3.826 \times 10^{-11}$ & $1.910 \times 10^{-10}$ & $1.540 \times 10^{-10}$ \\
\hline
\end{tabular}

Table 5. Results of ABAQUS for the constitutive equation (12) with default convergence criteria $R_{n}^{\alpha}=5 \times 10^{-3}$. The analytic solution for small strain is $\varepsilon=-1.1512 \times 10^{-3}$.

\begin{tabular}{|c||c|c|c||c|c|}
\hline \multicolumn{1}{|c||}{} & \multicolumn{3}{c||}{ small strains } & \multicolumn{2}{c|}{ large strains } \\
\cline { 2 - 6 } & $\begin{array}{c}\text { Forward Euler } \\
1000 \text { steps }\end{array}$ & $\begin{array}{c}\text { Extrap. Euler } \\
\text { TOL }=10^{-3}\end{array}$ & $\begin{array}{c}\text { Extrap. Euler } \\
\text { TOL }=10^{-10}\end{array}$ & $\begin{array}{c}\text { Forward Euler } \\
1000 \text { steps }\end{array}$ & $\begin{array}{c}\text { Extrap. Euler } \\
\text { TOL }=10^{-10}\end{array}$ \\
\hline$\varepsilon$ & $-1.1526 \times 10^{-3}$ & $-1.1519 \times 10^{-3}$ & $-1.1513 \times 10^{-3}$ & $-1.1526 \times 10^{-3}$ & $-1.1513 \times 10^{-3}$ \\
\hline CPU $[\mathrm{s}]$ & 1.91 & 0.37 & 183.53 & 1.91 & 183.46 \\
\hline
\end{tabular}

With the default convergence criteria ABAQUS needs 4 iterations in all cases. The solutions $\varepsilon$ are comparable, but the computational costs differ drastically, see Table 5.

The comparisons show clearly:

- The quadratic convergence plays only an important role when the residual is very small, normally smaller than engineers require (ABAQUS default: $R_{n}^{\alpha}=5 \times 10^{-3}$ ).

- The proposed method converges quadratically for high tolerances $\left(\mathrm{TOL}=10^{-10}\right)$. For lower tolerances $\left(\mathrm{TOL}=10^{-3}\right.$ ) we obtain a very rapid linear convergence which in practical applications cannot be distinguished from quadratical convergence.

- The computational costs are smaller for the extrapolated Euler, than for the forward Euler with constant step size and comparable accuracy.

- The use of constant step size without error control can lead to totally wrong results, see Table 1.

Next we study the same problem using hypoplasticity in the version of von Wolffersdorff [11]. To investigate the performance of the Jacobian calculation scheme, the convergence criterion for the ratio of the largest residuals to the corresponding average flux norm for convergence in the equilibrium Newton iteration was adjusted to $R_{n}^{\alpha}=10^{-8}$. The default value is $R_{n}^{\alpha}=5 \times 10^{-3}$. 
The decrease of the largest residual force during the equilibrium iterations listed in Table 6 shows the very rapid convergence. Only 5 iteration are needed to achieve the required accuracy. The calculation with the UMAT by Roddeman gives a much slower convergence and needs 37 equilibrium iterations.

Table 6. Largest residual force during equilibrium iterations of one-dimensional compression.

\begin{tabular}{|c|c|}
\hline \multicolumn{2}{|c|}{ proposed UMAT, TOL $=10^{-3}$} \\
\hline It.No. & largest residual force \\
\hline 1 & -67.1 \\
2 & 2.83 \\
3 & $6.447 \times 10^{-3}$ \\
4 & $5.218 \times 10^{-6}$ \\
5 & $3.187 \times 10^{-7}$ \\
\hline \hline CPU [s] & 0.50 \\
\hline
\end{tabular}

\begin{tabular}{|c|c|}
\hline \multicolumn{2}{|c|}{ UMAT - Roddeman } \\
\hline It.No. & largest residual force \\
\hline 33 & $-5.947 \times 10^{-6}$ \\
34 & $-3.576 \times 10^{-6}$ \\
35 & $-2.150 \times 10^{-6}$ \\
36 & $-1.293 \times 10^{-6}$ \\
37 & $-7.773 \times 10^{-7}$ \\
\hline \hline CPU [s] & 1.17 \\
\hline
\end{tabular}

To evaluate the computational costs, also calculations with 100 load increments and with the default convergence criterion was done. The results are shown in Tables 7 and 8. Although the computation of the Jacobian needs more effort in the 4 integration points, the overall calculation is faster in every investigated case. Also the unknown $\varepsilon_{22}$ of the problem is calculated more accurately.

Table 7. Comparison of computational costs with severer convergence criterion $R_{n}^{\alpha}=10^{-8}$ (one-dimensional hypoplastic compression).

\begin{tabular}{|l|c|c|c|c|c|c|}
\hline & \multicolumn{3}{|c|}{ 1 loading increment } & \multicolumn{3}{c|}{100 loading increments } \\
\hline UMAT & $\varepsilon_{22}$ & No. of It. & CPU [s] & $\varepsilon_{22}$ & No. of It. & CPU [s] \\
\hline proposed & $-1.1233 \times 10^{-3}$ & 5 & 0.50 & $-1.1231 \times 10^{-3}$ & 116 & 7.46 \\
Roddeman & $-1.1242 \times 10^{-3}$ & 37 & 1.17 & $-1.1231 \times 10^{-3}$ & 2394 & 49.01 \\
\hline
\end{tabular}

Table 8. Comparison of computational costs with default convergence criterion $R_{n}^{\alpha}=5 \times 10^{-3}$ (one-dimensional hypoplastic compression).

\begin{tabular}{|l|c|c|c|c|c|c|}
\hline & \multicolumn{3}{|c|}{1 loading increment } & \multicolumn{3}{c|}{100 loading increments } \\
\hline UMAT & $\varepsilon_{22}$ & No. of It. & CPU [s] & $\varepsilon_{22}$ & No. of It. & CPU [s] \\
\hline proposed & $-1.1233 \times 10^{-3}$ & 3 & 0.40 & $-1.1231 \times 10^{-3}$ & 102 & 5.26 \\
Roddeman & $-1.1336 \times 10^{-3}$ & 11 & 0.51 & $-1.1322 \times 10^{-3}$ & 132 & 5.61 \\
\hline
\end{tabular}

Note that in the cases with 100 load increments ABAQUS was not allowed to adjust the increments automatically, otherwise, it would increase the increments in calculations using the proposed UMAT because of the rapid convergence, as shown in the next example.

\subsection{Biaxial element test}

The next test is a biaxial element test as shown in Figure 3. The initial conditions are $T_{11}=T_{22}=$ $-p_{1}=-100 \mathrm{~N} / \mathrm{m}^{2}$, the initial void ratio is $e=0.735$. The loading is done by shifting the top nodes by $u_{2}=3 \times 10^{-3} \mathrm{~m}$.

ABAQUS was forced to start with a loading increment of $\Delta t=0.05$. The maximum allowed increment was $\Delta t=0.2$. The total loading time is 1.0. ABAQUS chose further load increments according to 


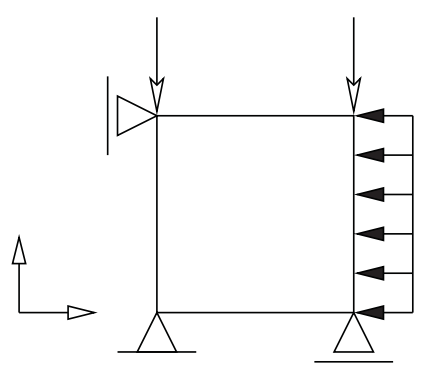

Figure 3. Biaxial element test.

the convergence of the equilibrium iteration. An exact solution was obtained with 1000 constant load increments, which gives the same solution for both subroutines.

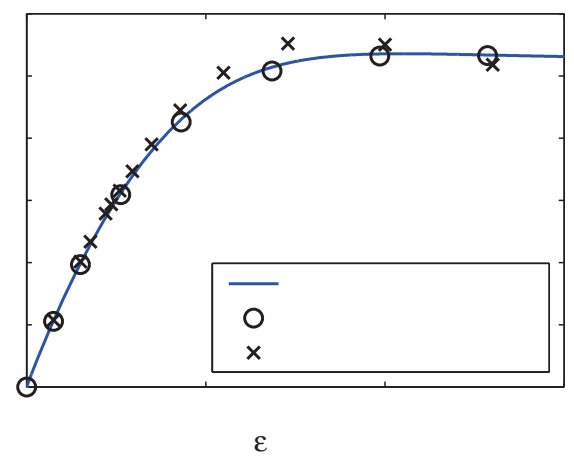

Figure 4. Stress strain curve: each circle and cross denotes an ABAQUS load increment for the calculations with the proposed UMAT and that of Roddeman, respectively.

Table 9. Comparison of the computational costs in the biaxial element test. The exact solution is: $T_{22}=-365.6$.

\begin{tabular}{|l|c|c|c|c|}
\hline \multicolumn{4}{|l|}{ ABAQUS: automatic load incrementation with $\Delta t_{\text {start }}=0.05, \Delta t_{\max }=0.2$} \\
\hline UMAT & $T_{22}\left[\mathrm{~N} / \mathrm{m}^{2}\right]$ & No. of Inc. & No. of It. & CPU [s] \\
\hline proposed & -365.7 & 8 & 15 & 0.9 \\
Roddeman & -360.9 & 15 & 41 & 1.37 \\
\hline
\end{tabular}

As shown in Table 9 and Figure 4, the calculations with the proposed UMAT are faster and more reliable.

\subsection{Simple shearing}

The last element test is a simple shear test as shown in Figure 5. The initial conditions are $T_{11}=$ $T_{22}=-p_{2}=-100 \mathrm{~N} / \mathrm{m}^{2}$, the initial void ratio is $e=0.735$. The loading is done by shifting the top nodes by $u_{1}=0.01 \mathrm{~m}$.

ABAQUS was forced to start with a loading increment of $\Delta t=0.05$. The maximum allowed increment was $\Delta t=0.2$. ABAQUS chose further load increments according to the convergence of the equilibrium iteration. An exact solution was obtained with 1000 constant load increments. 


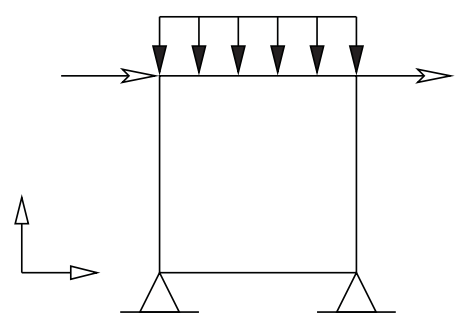

Figure 5. Simple-shear element test.

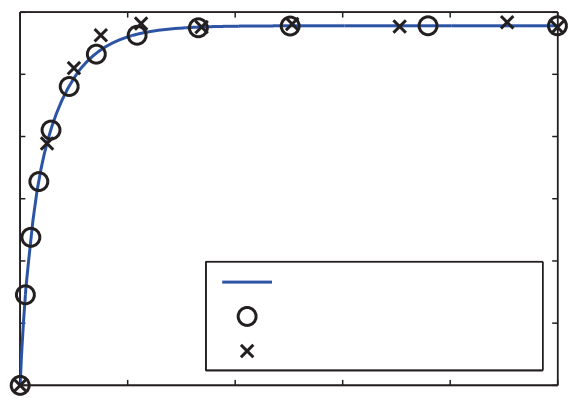

$\varepsilon$

Figure 6. Shear stress against shearing (circles and crosses denote ABAQUS load increments).

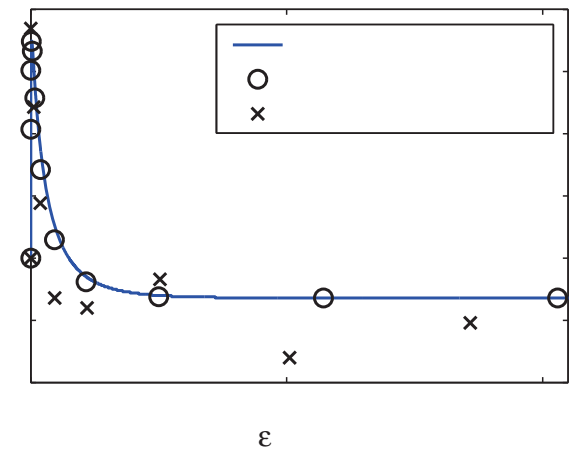

Figure 7. Horizontal stress against horizontal strain (circles and crosses denote ABAQUS load increments).

The used CPU time of the calculations with the proposed UMAT and that of Roddeman were almost the same, namely $0.97 \mathrm{~s}$ and $0.90 \mathrm{~s}$, respectively. As shown in Figures 6 and 7 the solution obtained with the proposed time integration scheme approximate much better the exact solution.

\subsection{Biaxial test}

This application is taken from Hügel [7]. A soil specimen of $0.04 \mathrm{~m}$ width and $0.14 \mathrm{~m}$ height is laterally compressed with a constant stress of size $4 \times 10^{5} \mathrm{~N} / \mathrm{m}^{2}$. The specimen is compressed vertically by a prescribed displacement $u=0.01 \mathrm{~m}$. The material in the dashed area of Figure 8 is given an initially higher void ratio of 0.45 , whereas the void ratio is 0.40 elsewhere. In this way, an initial imperfection in the dashed area is simulated. The dashed area is of the size $0.02 \mathrm{~m}$ by $0.02 \mathrm{~m}$.

The biaxial test is modeled with 8 by 28 linear plane strain elements. The calculation accounts for large strains and large rotations, so the lateral pressure remains perpendicular to the edge of the specimen. Two calculations were performed, one without gravity and one with gravity (specific weight of the sample $\gamma=17 \mathrm{kN} / \mathrm{m}^{3}$ ).

Figure 9 is a filled contour plot for the void ratio at the end of the test. This is plotted in the deformed piece of material. Note that the deformations are real and not scaled. It is clear that the void ratio did increase in the shear bands which are formed during the test. 


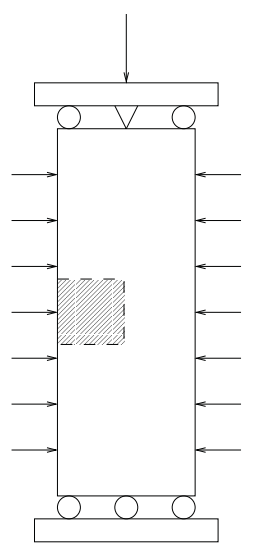

Figure 8. Biaxial test.
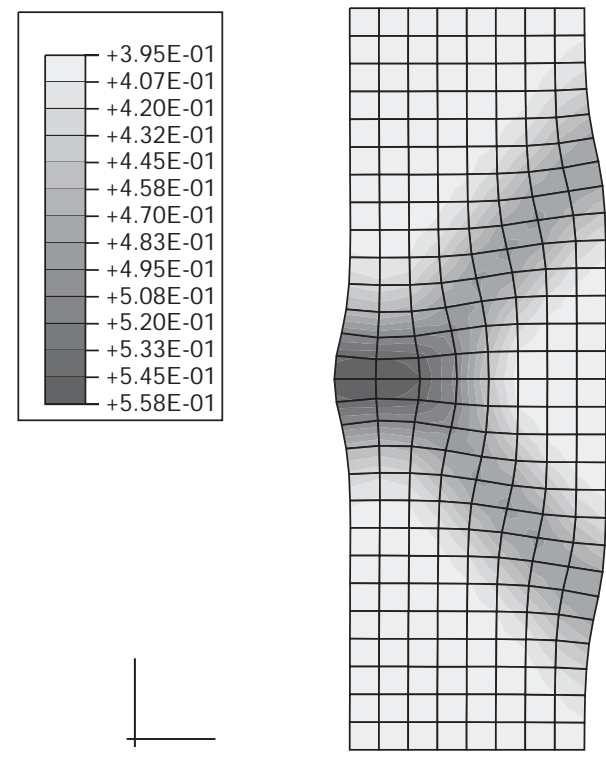

Figure 9. Biaxial compression test without gravity: void ratio; solution with proposed UMAT
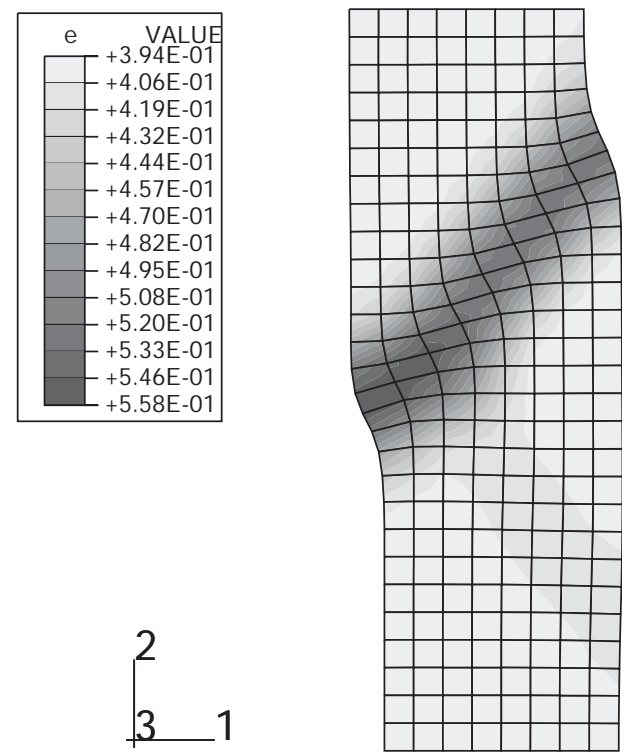

Figure 10. Biaxial compression test without gravity: void ratio; solution with UMAT Roddeman.

Figure 11 shows the size of the time steps used at the end of the test. Small time step sizes are required in the shear bands, whereas in other regions the time steps are much larger. This clearly indicates the adaptivity of our method.

The behaviour of the load incrementation strategy of ABAQUS is shown on the load displacement curve. The total loading time is $t=1$. The initial and the maximum load increments are $\Delta t=0.125$, so that at least 8 increments have to be done. In Figure 12 the exact solution (200 load increments) and the solution with automatic load incrementation are plotted. Each circle denotes a load increment.

One can see that in the loading branch the maximum allowed load increment was used, see Figure 12. Near the peak the load increment was decreased by the UMAT, then increased again by ABAQUS due to the rapid convergence. After the peak the load increments were decreased drastically, but even in the softening branch there was some increasing due to rapid convergence. In Figure 13 the load displacement curve obtained with the UMAT by Roddeman is shown. Due to the slower convergence the load increments chosen by ABAQUS are much smaller. Due to the very rough error control in 

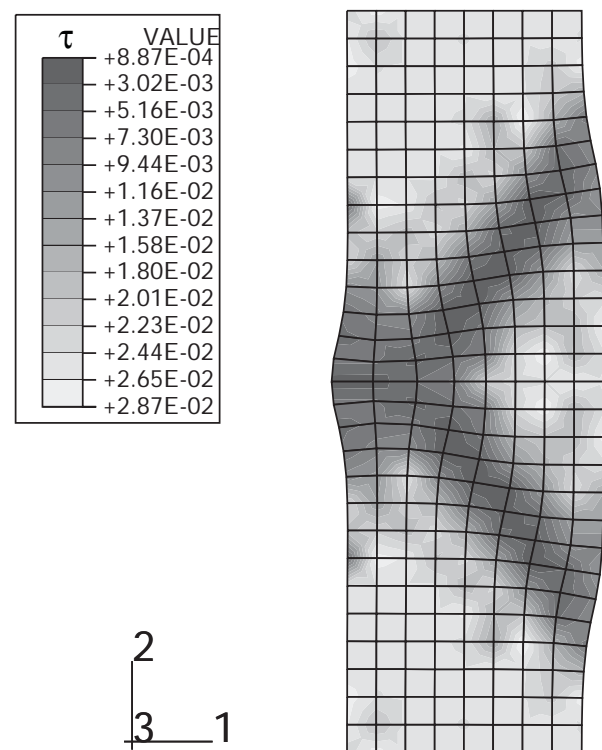

Figure 11. Biaxial compression test without gravity: time step size $\tau$ in UMAT.

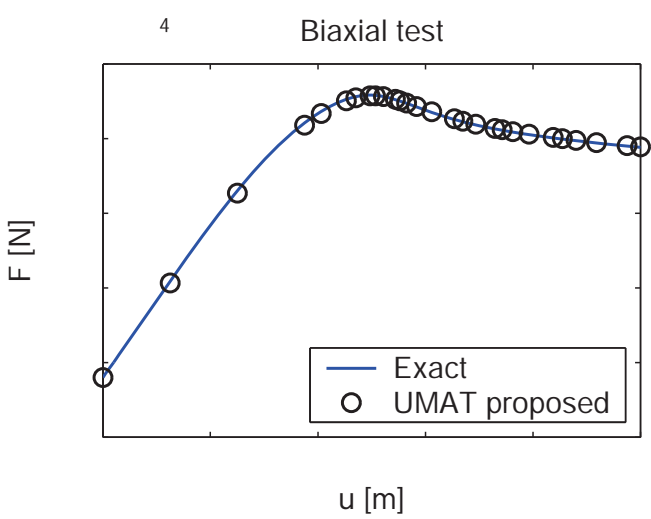

Figure 12. Biaxial compression test without gravity: Load displacement curve; UMAT proposed.

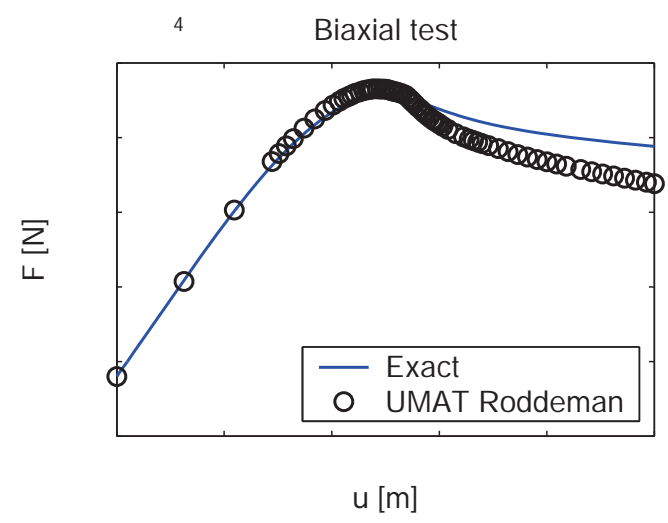

Figure 13. Biaxial compression test without gravity: Load displacement curve; UMAT Roddeman.

the old UMAT the post peak behaviour is much different, even only on shear band is formed, see Figure 10. The mesh dependence of the post peak behaviour is not studied here.

In the case of no gravity the problem is symmetric with respect to the horizontal direction, so the solution should show the same symmetry, see Figure 9. This is not the case for the solution obtained with the UMAT of Roddeman, see Figure 10. Since gravity destroys the vertical symmetry of the problem, only one shear band develops in calculations including gravity, see Figure 15. A shear band is more likely to develop downwards, because the stresses are higher at the bottom of the specimen due to the weight. Thus the initial state on the bottom of the specimen is nearer to the monotonically decreasing critical state line $e_{c}(\operatorname{tr} \mathbf{T})$ in the void-pressure diagram, see (23) in Appendix A. Due to the influence of gravity the system answer changes considerably, see Figure 14.

A comparison of the computational costs in Table 10 shows that the proposed UMAT is also more efficient. 


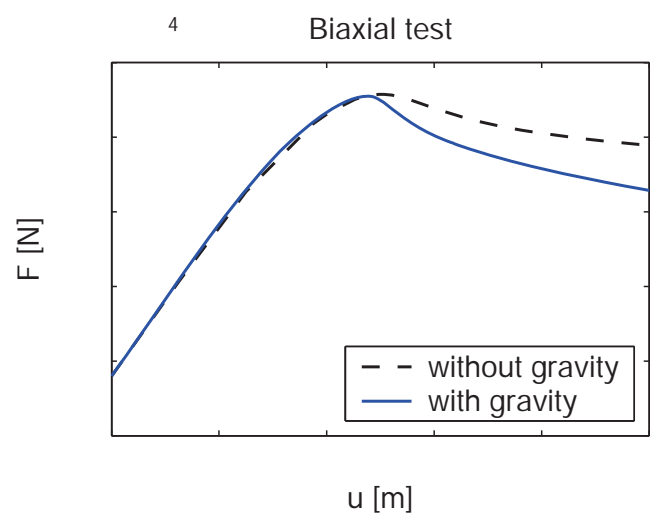

Figure 14. Biaxial compression test: Load displacement curve - UMAT proposed.
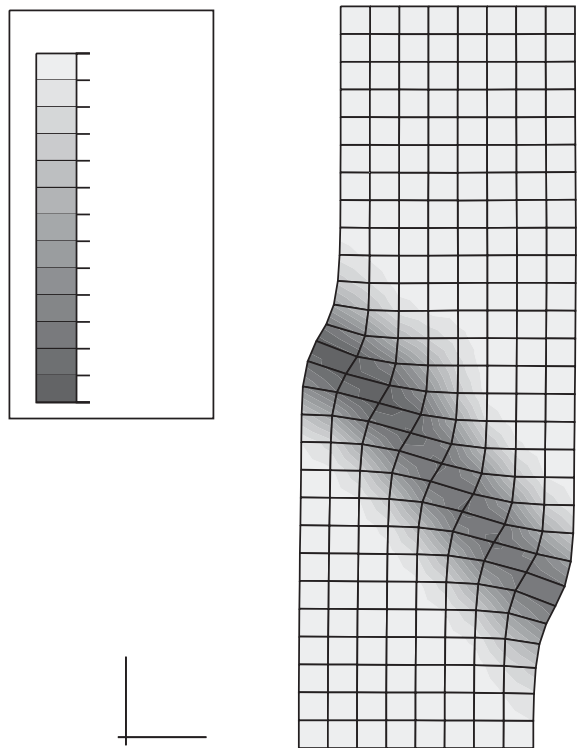

Figure 15. Biaxial compression test with gravity: void ratio; solution with proposed UMAT.

Table 10. Comparison of computational costs in the biaxial test without gravity. ABAQUS: automatic load incrementation with $\Delta t_{\text {start }}=0.125, \Delta t_{\max }=0.125$, and default convergence criteria for equilibrium iteration $R_{n}^{\alpha}=5 \times 10^{-3}$

\begin{tabular}{|l|c|c|c|}
\hline UMAT & No. of Inc. & No. of It. & CPU [s] \\
\hline proposed & 28 & 67 & 224.84 \\
Roddeman & 69 & 706 & 385.76 \\
\hline
\end{tabular}

\section{Conclusions}

In non-linear finite-element calculations the equations of motion and the non-linear constitutive law are solved separately in each loading increment. The subroutine which provides the constitutive law must compute the stresses at the end of the increment and the derivatives of the stresses with respect 
to the strain increments, the so called Jacobian. There are two crucial points in this calculation:

- An error control in time integration of the stress has to be provided. This is the basis for any adaptivity.

- The Jacobian has to be calculated in an accurate and flexible way, so that different constitutive laws can be implemented easily.

The above proposed scheme does both jobs. Quadratic convergence of the equilibrium Newton iteration in an implicit finite-element calculation was shown, using the incrementally non-linear hypoplastic constitutive law. We emphasize, that our approach is sufficiently general to cover all constitutive laws in rate form, e.g. the CloE-model [6].

\section{Acknowledgment}

We thank the anonymous referees for valuable suggestions that helped to improve the presentation of the paper.

\section{References}

[1] J.C. Simo and T.J.R. Hughes. Computational Inelasticity. Springer, 1998.

[2] C. Miehe. Numerical computation of algorithmic (consistent) tangent moduli in large-strain computational inelasticity. Computer methods in applied mechanics and engineering 1996; 134:223-240.

[3] A. Pérez-Foguet, A. Rodríguez-Ferran and A. Huerta. Numerical differentiation for local and global tangent operators in computational plasticity. Computer methods in applied mechanics and engineering 2000; 189:277-296.

[4] A. Pérez-Foguet, A. Rodríguez-Ferran and A. Huerta. Numerical differentiation for non-trivial consistent tangent matrices: an application to the MRS-lade model. International Journal for Numerical Methods in Engineering 2000; 48:159-184.

[5] A. Pérez-Foguet, A. Rodríguez-Ferran and A. Huerta. Consistent tangent matrices for densitydependent finite plasticity models. International Journal for Numerical and Analytical Methods in Geomechanics 2001; 25:1045-1075.

[6] J. Desrues and R. Chambon. A new rate type constitutive model for geomaterials. In Modern Approaches to Plasticity, D. Kolymbas (ed). Elsevier, 1993; 309-324

[7] H. Hügel. Prognose von Bodenverformungen. Veröffentlichung des Institutes für Bodenmechanik und Felsmechanik der Universität Fridericiana in Karlsruhe, Heft 136, 1995.

[8] D. Roddeman. FEM-implementation of hypoplasticity. European Union Projekt (ERBCHBGCT 940554), Institut für Geotechnik und Tunnelbau, Baufakultät, Universität Innsbruck, 1997. 
[9] O.M. Heeres. Modern Strategies for the Numerical Modeling of the Cyclic and Transient Behavior of Soils. PhD thesis, TU Delft, DUP Science - Delft University Press, 2001.

[10] C. Truesdell and W. Noll. The Non-Linear Field Theories of Mechanics. Springer, 1965.

[11] P.-A. von Wolffersdorff. A hypoplastic relation for granular materials with a predefined limit state surface. Mechanics of Cohesive-Frictional Materials 1996; 1:251-271.

[12] D. Kolymbas. Introduction to Hypoplasticity. Advances in Geotechnical Engineering and Tunnelling 1. Balkema, 2000.

[13] A. Niemunis and I. Herle. Hypoplastic model for cohesionless soils with elastic strain range. Mechanics of Frictional and Cohesive Materials 1997; 2:279-299.

[14] Abaqus. Theory manual, Version 5.8. HKS Inc., Hibbit, Karlson \& Sorenson: Rhode Island, 1998.

[15] Abaqus. User's Manual, Version 5.8, Volume 3. HKS Inc., Hibbit, Karlson \& Sorenson: Rhode Island, 1998.

[16] E. Hairer, S.P. Nørsett, and G. Wanner. Solving Ordinary Differential Equations I. Nonstiff Problems. 2nd edition, Springer: Berlin, 1993.

[17] W. Fellin and A. Ostermann. A hypoplasticity routine for ABAQUS with consistent tangent operator and error control. http://geotechnik.uibk.ac.at/res/FEhypo.html, 2001.

[18] O.M. Heeres and R. de Borst. Implicit integration of hypoplastic models. In Constitutive Modelling of Granular Materials, D. Kolymbas (ed). Springer, 2000; 457-470

[19] W. Fellin. Hypoplastizität für Einsteiger. Bautechnik 2000; 77(1):10-14.

[20] I. Herle. Hypoplastizität und Granulometrie einfacher Korngerüste. Heft 151, Institut für Bodenmechanik und Felsmechanik, Universität Karlsruhe, 2000.

\section{A Hypoplastic basic model}

For the sake of completeness, we outline the full hypoplastic model as it was proposed in [11]

$$
\stackrel{\circ}{\mathbf{T}}=\mathcal{L}(\mathbf{T}, e): \mathbf{D}+\mathbf{N}(\mathbf{T}, e)\|\mathbf{D}\|,
$$

with the linear term

$$
\mathcal{L}=f_{s} \frac{1}{\operatorname{tr}\left(\hat{\mathbf{T}}^{2}\right)}\left(F^{2} \mathbf{I}+a^{2} \hat{\mathbf{T}}^{2}\right)
$$

and the non-linear term

$$
\mathbf{N}=f_{s} f_{d} \frac{a F}{\operatorname{tr}\left(\hat{\mathbf{T}}^{2}\right)}\left(\hat{\mathbf{T}}+\hat{\mathbf{T}}^{*}\right)
$$


The used stress variables are defined as follows

$$
\hat{\mathbf{T}}:=\frac{\mathbf{T}}{\operatorname{tr} \mathbf{T}}, \quad \hat{\mathbf{T}}^{*}:=\hat{\mathbf{T}}-\frac{1}{3} \mathbf{I} .
$$

The factors for pressure and density dependency (barotropy and pyknotropy) are given by

$$
\begin{aligned}
a & :=\frac{\sqrt{3}\left(3-\sin \varphi_{c}\right)}{2 \sqrt{2} \sin \varphi_{c}}, \quad f_{d}:=\left(\frac{e-e_{d}}{e_{c}-e_{d}}\right)^{\alpha} \\
f_{s} & :=\frac{h_{s}}{n}\left(\frac{e_{c}}{e}\right)^{\beta} \frac{1+e_{i}}{e_{i}}\left(\frac{-\operatorname{tr} \mathbf{T}}{h_{s}}\right)^{1-n}\left[3+a^{2}-a \sqrt{3}\left(\frac{e_{i 0}-e_{d 0}}{e_{c 0}-e_{d 0}}\right)^{\alpha}\right]^{-1}
\end{aligned}
$$

The factor $F$ for adapting the deviatoric yield curve to Matsuoka-Nakai is

$$
F:=\sqrt{\frac{1}{8} \tan ^{2} \psi+\frac{2-\tan ^{2} \psi}{2+\sqrt{2} \tan \psi \cos 3 \theta}}-\frac{1}{2 \sqrt{2}} \tan \psi
$$

with

$$
\tan \psi:=\sqrt{3}\left\|\hat{\mathbf{T}}^{*}\right\| \quad \text { and } \quad \cos 3 \theta:=-\sqrt{6} \frac{\operatorname{tr}\left(\hat{\mathbf{T}}^{3}\right)}{\left[\operatorname{tr}\left(\hat{\mathbf{T}}^{2}\right)\right]^{3 / 2}}
$$

The void ratios must fulfill the compression law

$$
\frac{e_{i}}{e_{i 0}}=\frac{e_{c}}{e_{c 0}}=\frac{e_{d}}{e_{d 0}}=\exp \left[-\left(\frac{-\operatorname{tr} \mathbf{T}}{h_{s}}\right)^{n}\right] .
$$

This hypoplastic law has 8 parameters: the critical friction angle $\varphi_{c}$, the granular hardness $h_{s}$, the void ratios $e_{i 0}, e_{c 0}$ and $e_{d 0}$, and the exponents $n$ and $\beta$. They can be easily determined from simple index and element tests [20].

The hypoplastic parameters used in all calculations are listed in Table 11.

Table 11. Parameters for the von Wolffersdorff hypoplastic law.

\begin{tabular}{|c|c|c|c|c|c|c|c|}
\hline$\varphi_{c}$ & $h_{s}$ & $e_{c 0}$ & $e_{d 0}$ & $e_{i 0}$ & $n$ & $\alpha$ & $\beta$ \\
\hline $30^{\circ}$ & $0.19 \times 10^{9} \mathrm{~N} / \mathrm{m}^{2}$ & 0.8 & 0.4 & 1.189 & 0.45 & 0.15 & 1.0 \\
\hline
\end{tabular}

